\title{
Patient perception of smartphone usage by doctors
}

\author{
Georgina Kerry' \\ Shyam Gokani \\ Dara Rasasingam² \\ Alexander Zargaran ${ }^{3}$ \\ Javier Ash ${ }^{2}$ \\ Aaina Mittal ${ }^{2}$ \\ 'College of Medical and Dental \\ Sciences, University of Birmingham, \\ Birmingham, ${ }^{2}$ Faculty of Medicine, \\ Imperial College London, London, \\ 'Faculty of Medicine, St George's \\ University of London, London, UK
}

Correspondence: Georgina Kerry College of Medical and Dental Sciences, University of Birmingham, Edgbaston,

Birmingham, BI5 2TT, UK

Tel +44078 53862253

Email gxk14I@student.bham.ac.uk
This article was published in the following Dove Press journal:

Smart Homecare Technology and TeleHealth

6 March 2017

Number of times this article has been viewed

\begin{abstract}
Technological advancements have revolutionized modern medicine and smartphones are now ubiquitous among health care professionals. The ability to look up information promptly is invaluable to doctors and medical students alike, but there is an additional contiguous benefit to patients. Queries can be answered more accurately through fingertip access to evidencebased medicine, and physicians have instant access to emergency care protocols. However, is consideration always extended to the patient's perception of the use of smartphones by doctors? Do patients know why we use smartphones to assist us in their care? What do they think when they see a doctor using a smartphone?

An independent question, conducted within a wider service evaluation (ethical approval not required, full verbal and written electronic consent provided by all patients) at St. Mary's Hospital, London, indicated that although the majority $(91.0 \%)$ of patients owned a smartphone, many (61.6\%) did not agree that the use of smartphones at work by doctors is professional. This highlights the potential for damage to the doctor-patient relationship. There is a risk that these patients will disconnect with care services with possible detriment to their health. Additionally, it is notable that a larger proportion of those patients aged $>70$ years found the use of smartphones by doctors at work unprofessional, compared with patients aged $<70$ years.

Adequate communication between the doctor and patient is critical in ensuring that doctors can make use of modern technology to provide the best possible care and that patients are comfortable with this and do not feel isolated or consider the doctor ignorant. It is suggested that moves are made to educate patients of the importance of the use of technology by doctors and to ensure that patients are aware of the reasons for which doctors use smartphones at work.
\end{abstract}

Keywords: telehealth, smartphone, doctor-patient relationship, patient self-determination act

Technological advancements have revolutionized modern medicine. Robot-assisted surgery has drastically enhanced a surgeon's performance, ${ }^{1}$ while the automation of biochemical analysis and the recording of observations attempts to eliminate the potential for human error. While there is no doubt that technology improves clinical outcomes for patients on the whole, we question whether or not this aligns positively with holistic, patient-centered care.

Smartphones are ubiquitous among health care professionals, and the number of health care applications (apps) available for clinical use is fast growing. As final year medical students, we are encouraged by clinical tutors to familiarize ourselves with 
features such as electronic prescribing systems and picture archiving and communication system. More recently though is also the suggestion of helpful apps, such as the British National Forumulary (BNF) smartphone application, and trust-specific apps to assist with drug choice and provide trust-specific management protocols.

For the doctor or student, the ability to look up information promptly is invaluable, and the response to an emergency situation is greatly improved by instantaneous access to appropriate guidelines. There is additionally a contiguous benefit to the patient in the reduction of any delay to treatment and the ability to answer queries more accurately through fingertip access to evidence-based medicine.

However, is consideration always extended to the patient's perception of the use of smartphones in clinic or at bedside? Sixty-eight percent of UK adults own a smartphone $^{2}$ and are, therefore, likely to know of the potentials of their use in assisting clinical diagnosis and management. They are, however, unlikely to be aware of the availability of applications specific to educating and assisting medical professionals and may also be unaware of the variation in management protocols between trusts and clinical situations. Therefore, the impact of the use of smartphones by health care professionals on the doctor-patient relationship is brought into question.

The doctor-patient relationship is founded in trust, and patients may perceive the use of a smartphone by the doctor as distraction or disinterest, decreasing the level of trust in the relationship and harming the development of a rapport. Many patients are of older age and it is known that the prevalence of smartphone ownership is considerably lower among this age group. ${ }^{3}$ These patients are, therefore, those most at risk of misperceiving the use of smartphones by doctors and experiencing damage to the doctor-patient relationship.

A recent service evaluation conducted at St. Marys Hospital, London, of 401 bariatric surgical patients indicated that although $91.0 \%$ owned a smartphone themselves, only $38.4 \%$ did not agree with the statement: "Doctors and nurses should not use smartphone or tablet devices while at work because this is unprofessional" (Figure 1), indicating that most patients consider the use of smartphones by doctors as unprofessional. Patients are unsure about the use of smartphones by doctors and may incorrectly perceive their use for clinical assistance as unprofessional, personal use. The opinion also varied based upon the age of the patients: $40 \%$ of those under the age of 70 years disagreed with the statement that the use of smartphones at work by doctors was unprofessional, compared with $12 \%$ of those over the age of 70 years $\left(\chi^{2}=48.3\right.$, df: $28, p=0.01$; Figure 2$)$.

Thorough emphasis is placed on the development of excellent communication skills in medical students as the development of the doctor-patient relationship relies on these skills. Additionally, patient satisfaction is impacted by the quality of a clinician's communication skills. The use of a smartphone may be perceived by the patient as unprofessional or ignorant, which in turn decreases patient satisfaction and proves detrimental to the doctor-patient relationship.

Furthermore, Neilsen's system acceptability model states that an IT system is only accepted where it is both socially and practically acceptable. The use of smartphones by

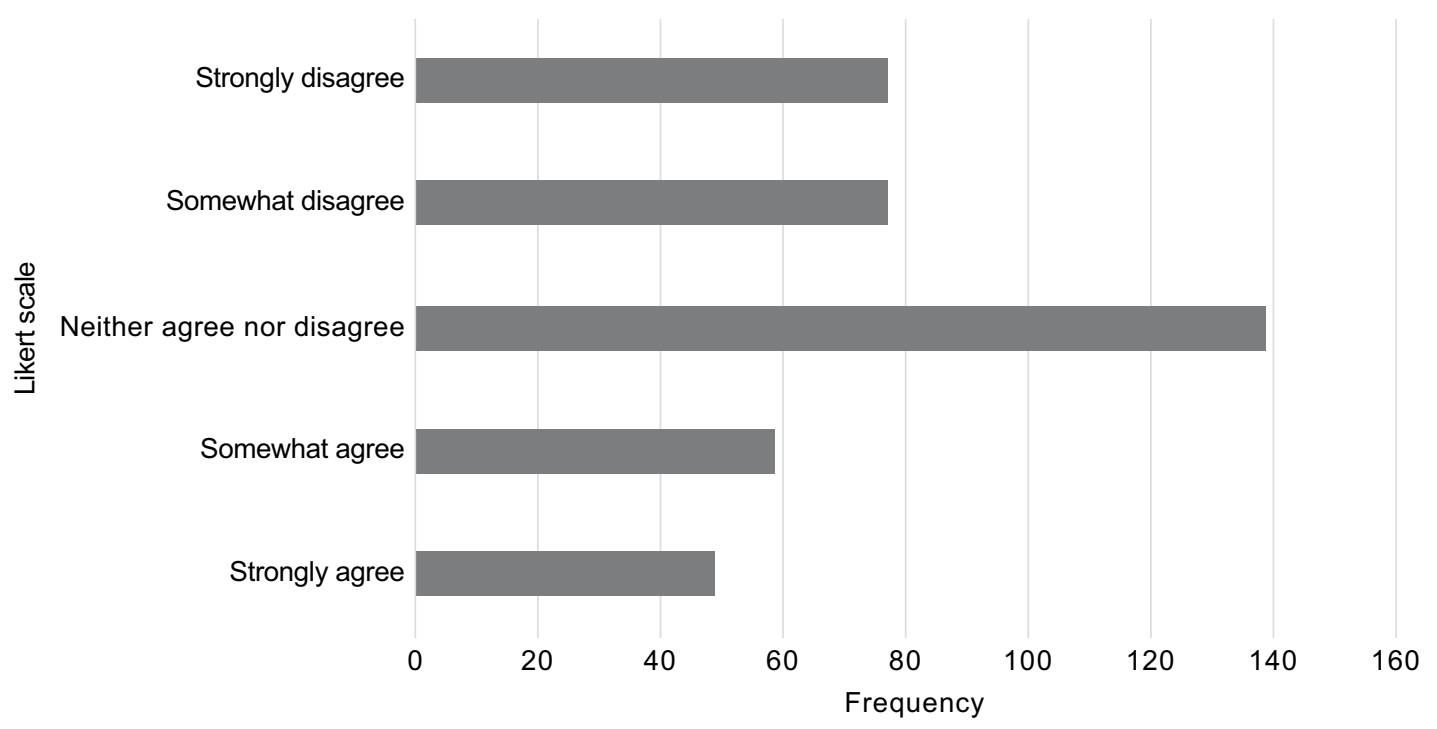

Figure I Bar chart to show answers for the question: "To what extent do you agree with the statement: 'Doctors and nurses should not use smartphone or tablet devices at work because this is unprofessional'?" 


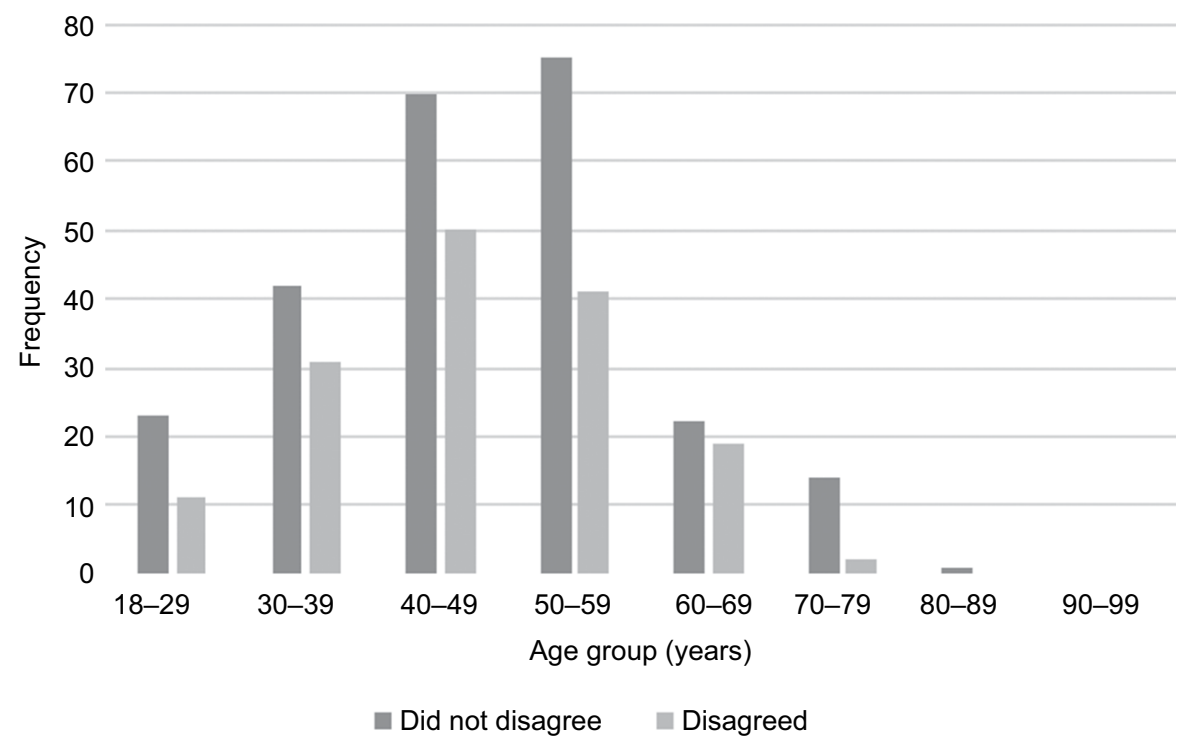

Figure 2 Bar chart to show the age range for answers to the question: "To what extent do you agree with the statement "Doctors and nurses should not use smartphone or tablet devices at work because this is unprofessional'?"

doctors may not be deemed socially acceptable by patients and, therefore, difficulties during consultation and care may be encountered.

The lack of clarity surrounding the purpose of the use of smartphones/tablets by doctors in this independent question generates limitations in the reliability of the data collected. It was informally noted by data collectors that occasionally patients mentioned that it "depended" what the doctor was using their smartphone for. However, this highlights the need for clarification by health care professionals of the purpose of their use of smartphone/tablet devices during working hours. As previously alluded to, clear communication between patient and doctor is critical in the maintenance of a good doctor-patient relationship.

An additional limitation is that this study only looks at bariatric patients and, therefore, its applicability to generic patient populations is questioned. For example, these patients were outpatients, and the experience of an inpatient may be different as they can observe doctors and health care professionals carrying out daily tasks and directly interact with them. In outpatient clinics, mainly direct interaction occurs.

We believe that doctors and students should continue to use smartphone apps to support their education and clinical practice as their usefulness cannot be ignored. However, we additionally suggest that the importance of developing trust and rapport between is not forgotten as the provision of a good history relies upon the quality of this doctor-patient relationship. Further research into patients' perceptions of smartphone use is warranted in order to appropriately direct education and to fully inform patients on the reasons for their use. Where smartphones are used in the presence of patients, their use should be explained and agreed with the patient wherever possible. Efforts can be made to educate patients on the usefulness of smartphones to a clinician's practice in order to preserve rapport.

\section{Ethical approval}

This study uses data collected as part of a wider service evaluation and, therefore, ethical approval was not required. All patients provided verbal and written electronic consent.

\section{Disclosure}

The authors report no conflicts of interest in this work.

\section{References}

1. Cao CGL, Rogers G. Robotics in Healthcare: HF Issues in Surgery. Boston: Tufts University; 2006.

2. Poushter J. Smartphone Ownership and Internet Usage Continues to Climb in Emerging Economies. 2016. Available from: http://www. pewglobal.org/2016/02/22/smartphone-ownership-and-internet-usagecontinues-to-climb-in-emerging-economies/. Accessed September 19, 2016.

3. Statista. UK Smartphone Ownership by Age from 2012-2016. Available from: https://www.statista.com/statistics/271851/smartphone-ownersin-the-united-kingdom-uk-by-age/. Accessed September 19, 2016. 


\section{Publish your work in this journal}

Smart Homecare Technology and TeleHealth is an international, peer-reviewed, open access online journal publishing original research, reviews, editorials and commentaries on the application of technology to support people and patients at home and in assisted living centers to optimize healthcare and management resources. Specific topics in the journal include: Development and application of devices within the home and embedded in appliances; Healthcare provider communication and education tools; and drug ordering and adherence. The manuscript management system is completely online and includes a very quick and fair peer-review system, which is all easy to use. Visit http://www.dovepress.com/ testimonials.php to read real quotes from published authors.

Submit your manuscript here: https://www.dovepress.com/smart-homecare-technology-and-telehealth-journal 